\title{
Convergent-close-coupling formalism for positron scattering from molecules
}

\author{
Mark C. Zammit, 'Dmitry V. Fursa, and Igor Bray \\ ARC Centre for Antimatter-Matter Studies, Curtin University, GPO Box U1987, Perth, Western Australia 6845, Australia
}

(Received 8 December 2012; published 7 February 2013)

\begin{abstract}
The $a b$ initio convergent-close-coupling method has been extended to positron-molecule collisions within the adiabatic (fixed-nuclei) approximation. Application to molecular hydrogen at energies from 0.1 to $1000 \mathrm{eV}$ has yielded convergent total ionization and grand total cross sections over most of the energy range. We find that very large calculations are required for convergence, even in the case of low-energy elastic scattering, due to the effects of positronium formation. In general, the comparison with experiment is good.
\end{abstract}

DOI: 10.1103/PhysRevA.87.020701

PACS number(s): $34.80 . \mathrm{Uv}, 34.50 . \mathrm{Fa}$, 52.20.Fs

The convergent-close-coupling (CCC) method has achieved considerable success in providing accurate collision data for electron and positron scattering from atoms and ions [1-3]. The fundamental strength of the method is its ability to account for coupling to the ionization channels [4]. The target continuum is incorporated to convergence utilizing pseudostates obtained via diagonalization of the target Hamiltonian in a complete Sturmian (Laguerre) basis. We suggest that the CCC implementation for atoms is a complete scattering theory in the sense that it yields accurate elastic, excitation, and ionization cross sections irrespective of the projectile energy [5,6]. Our long-term goal is to do the same for molecules. Here, we report the first step, the implementation of the CCC method to molecular hydrogen, in the context of a single-center approach to positron collisions.

Theoretical approaches to positron scattering with molecules have traditionally focused on the low-energy region, where the $R$ matrix [7,8], Schwinger multichannel [9], and complex Kohn variational [10] methods have been applied with considerable success. Also, the single-channel complex model potential method of [11] produced some good results at low and intermediate energies. However, to date, no ab initio methods have been applied over the entire energy region of interest for any molecule.

Positron collisions with atoms and molecules present a set of theoretical challenges that are particularly difficult due to the inherently multicenter nature of the collision problem. One center is the center of mass of the target, and the other is the center of mass of positronium (Ps). Furthermore, the multicenter nature of molecular wave functions leads to a separate set of problems such as the absence of spherical symmetry, and the appearance of new reaction channels related to rotational and vibrational excitations and dissociative recombination and attachment processes.

Fortunately, the single-center CCC approach to positron scattering is as valid as the two-center one at energies outside the small energy region between the Ps-formation and direct ionization thresholds. This is because at energies above the ionization threshold, the positive-energy pseudostates collectively take into account both breakup and Ps formation. At energies below Ps formation, the pseudostates take into account virtual atomic excitation and Ps formation. Consequently, the single-

*mark.zammit@postgrad.curtin.edu.au center CCC approach is an ideal starting point, with the complicated addition of explicit Ps formation to be done at a later stage; see [12-14] for two-center approaches to positron scattering on $\mathrm{H}, \mathrm{He}$, and $\mathrm{Li}$, respectively.

Extension of the CCC method to molecular hydrogen requires a set of target (pseudo)states obtained from a complete basis. We adopt the fixed-nuclei approximation and perform calculations at the ground-state equilibrium internuclei distance, which is $R=1.4 a_{0}$. The target electronic Hamiltonian is

$$
H_{\mathrm{T}}=H_{1}+H_{2}+V_{12}+1 / R
$$

where $1 / R$ is the internuclear Coulomb repulsion, $V_{12}$ is the interaction between the two electrons,

$$
H_{i}=-\frac{1}{2} \nabla_{i}^{2}+V\left(\boldsymbol{r}_{i}, \boldsymbol{R}\right), \quad i=1,2,
$$

and the electron-nuclei potential is given by

$$
V\left(\boldsymbol{r}_{i}, \boldsymbol{R}\right)=-\frac{1}{\left|\boldsymbol{r}_{i}-\boldsymbol{R} / 2\right|}-\frac{1}{\left|\boldsymbol{r}_{i}+\boldsymbol{R} / 2\right|} .
$$

The origin of the body-frame coordinate system is at the center of mass of the molecule and the $z$ axis is aligned with $\boldsymbol{R}$. Atomic units are used throughout the paper.

The target Hamiltonian (1) is diagonalized in a set of antisymmetrized two-electron configurations, built from Laguerre one-electron orbitals, for each target symmetry characterized by the projection of orbital angular momentum $m_{\mathrm{T}}$, parity $\pi_{\mathrm{T}}$, and spin $s_{\mathrm{T}}$. For positron scattering from the ground state of $\mathrm{H}_{2}$, only states with $s_{\mathrm{T}}=0$ are required. The $N$ molecular target states satisfy

$$
\left\langle\Phi_{i}^{(N)}\left|H_{\mathrm{T}}\right| \Phi_{j}^{(N)}\right\rangle=\varepsilon_{j}^{(N)} \delta_{i j}
$$

The lack of spherical symmetry leads to a substantial increase in the number of states in the close-coupling expansion compared to the atomic case. This is particularly problematic for positron scattering as states with large $m_{\mathrm{T}}$ are required for a convergent treatment of Ps formation.

The momentum-space close-coupling method, on which the CCC method is based, is particularly suited to treating large close-coupling expansions. The total wave function is substituted into the body-frame Schrödinger equation and then transformed to a set of coupled Lippmann-Schwinger 
equations for the $T$ matrix,

$$
\begin{aligned}
\left\langle\boldsymbol{k}_{f} \Phi_{f}^{(N)}\left|T^{(N)}\right| \Phi_{i}^{(N)} \boldsymbol{k}_{i}\right\rangle & \\
= & \left\langle\boldsymbol{k}_{f} \Phi_{f}^{(N)}|V| \Phi_{i}^{(N)} \boldsymbol{k}_{i}\right\rangle \\
& +\sum_{n=1}^{N} \int d k \frac{\left\langle\boldsymbol{k}_{f} \Phi_{f}^{(N)}|V| \Phi_{n}^{(N)} \boldsymbol{k}\right\rangle\left\langle\boldsymbol{k} \Phi_{n}^{(N)}\left|T^{(N)}\right| \Phi_{i}^{(N)} \boldsymbol{k}_{i}\right\rangle}{E^{(+)}-k^{2} / 2-\varepsilon_{n}^{(N)}} .
\end{aligned}
$$

This is solved by performing a partial-wave expansion of the positron plane waves $|\boldsymbol{k}\rangle$ to obtain $T$-matrix elements per partial wave of total angular projection $M$, spin $S$, and parity $\Pi$. The $T$-matrix elements are then used to obtain body-frame scattering amplitudes $f_{\mathrm{fi}}^{(\mathrm{B})}$. An analytic Born subtraction method [15] is employed to reduce the number of partial waves requiring explicit solution.

In order to account for the random orientation of the molecular target, we have utilized orientation averaging procedures [15] to obtain integrated and differential cross sections (DCS). To achieve this, $f_{\mathrm{fi}}^{(\mathrm{B})}$ are transformed to the laboratory-frame scattering amplitudes $f_{\mathrm{fi}}^{(\mathrm{L})}$ via rotation by Euler angles $\boldsymbol{\beta}$, which orientate the body frame relative to the laboratory frame. The DCS are defined by averaging over all orientations of the molecule,

$$
\frac{d \sigma_{\mathrm{fi}}}{d \Omega}=\frac{1}{8 \pi^{2}} \int\left|f_{\mathrm{fi}}^{(\mathrm{L})}(\boldsymbol{\beta})\right|^{2} d \boldsymbol{\beta} .
$$

As a part of demonstrating convergence, calculations have been performed for a number of structure models that differ in value of maximum orbital angular momentum $l_{\max }$ of the Laguerre basis. The Laguerre basis used $10-l$ functions for $l<5$ and five for $l \geqslant 5$. All functions were chosen with an exponential falloff of 1.9. The Laguerre basis $1 s$ orbital was replaced with the $\mathrm{H}_{2}^{+} 1 s \sigma_{g}$ state, which is obtained via diagonalization of the $\mathrm{H}_{2}^{+}$Hamiltonian using a large Laguerre basis (with $l_{\max }^{\prime}=8$ and number of orbitals increased to convergence). This choice of $1 \mathrm{~s}$ orbital is particularly important to model the continuum spectrum of the $\mathrm{H}_{2}$ molecule with positive-energy pseudostates. The two-electron configurations have been selected according to the following rules: all frozen-core type configurations $(1 s, n l m)$ were included and, in addition, all $\left(n^{\prime} l^{\prime} m^{\prime}, n l m\right)$ configurations were included for Laguerre orbitals with principle quantum numbers $n^{\prime}$ and $n \leqslant 3$. All states obtained in the diagonalization procedure have been included in the close-coupling expansion. This includes all $\mathrm{H}_{2}$ target states with the maximum value of orbital angular momentum projection $\left|m_{\mathrm{T}}\right|$ to be equal to $l_{\max }$.

The largest model for which calculations have been performed across the whole energy range has $l_{\max }=8$ and contains $N=566$ states. With this basis, the ground-state energy is -1.1687 , which compares well with the accurate value of -1.1745 [16]. The ground-state static dipole polarizabilities are $\alpha_{\perp}=4.6413$ and $\alpha_{\|}=6.3885$, which are both in good agreement with the accurate calculations of Kolos and Wolniewicz [17] $\left(\alpha_{\perp}=4.5777\right.$ and $\left.\alpha_{\|}=6.3805\right)$.

The convergence of the calculations for the grand total cross section (GTCS) on the energy range from 0.1 to $1000 \mathrm{eV}$ is demonstrated in Fig. 1. Calculations have been performed with $l_{\max }=2(N=165)$ through to $l_{\max }=8(N=556)$. In the

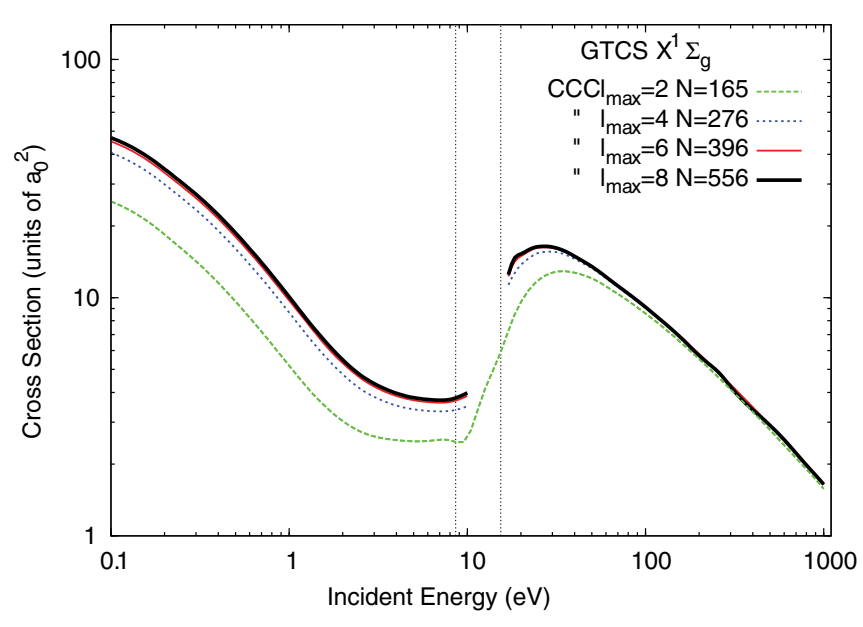

FIG. 1. (Color online) Convergence studies of the grand total cross section (GTCS) for positron scattering from the ground state of $\mathrm{H}_{2}$. The $\mathrm{CCC}$ calculations are labeled by basis functions with maximum angular momentum $l_{\max }$ and the resultant number of states $N$. The two vertical lines indicate the Ps-formation and ionization thresholds.

low-energy region, convergence is reached for the $l_{\max }=6 \mathrm{cal}$ culations. Note the large difference between the $l_{\max }=2$ and other, larger $l_{\max }$ calculations. This indicates the importance of the coupling to virtual Ps-formation channels, as opposed to just accounting for the correct static dipole polarizability. In the region between Ps-formation and ionization thresholds, a single-center model often shows large cross-section spikes due to a mismatch in boundary conditions (open Ps-formation channels and closed ionization channels). The results with $l_{\max } \geqslant 4$ in this region have been excluded. The $l_{\max }=2$ model has few Ps-formation channels, and as a result yields a reasonable, though not necessarily accurate, GTCS in this region. For energies above the direct ionization threshold, the GTCS converges most slowly to the cross-section maximum at $30 \mathrm{eV}$. This is the energy region where the Ps formation is at a maximum. In the single-center method, the latter is modeled as coupling to large $m_{\mathrm{T}}$ positive-energy pseudostates. As the impact energy increases, coupling to Ps-formation channels becomes progressively less important and convergence can be achieved with smaller $l_{\max }$.

At the very low energies, the GTCS and the $s$-wave phase shift are related to the scattering length $A$ as $\sigma(0)=4 \pi A^{2}$ and $\tan \delta_{0}=-A k$ [18]. We have conducted calculations to sufficiently low energies to produce an estimate for the scattering length $A=-2.49 a_{0}$, which is in good agreement with recent calculation of Zhang et al. [19], who obtain $A=$ $-2.61 a_{0}$ (calculated from the soft-box radius with internuclei distance $\left.R=1.4 a_{0}\right)$.

At energies below the Ps-formation threshold, there is very good agreement between the $l_{\max }=8 \mathrm{CCC}$ results and a number of previous theoretical models. These include the $R$ matrix with pseudostates (MRMPS) calculations of Zhang et al. [7], Schwinger multichannel calculations of Arretche et al. [9], and various first-order methods [20,21]. For clarity of presentation, in Fig. 2 we compare only with the MRMPS results, which are marginally below the $\mathrm{CCC}$ results. The difference is most likely due to the fact that the MRMPS calculations had 


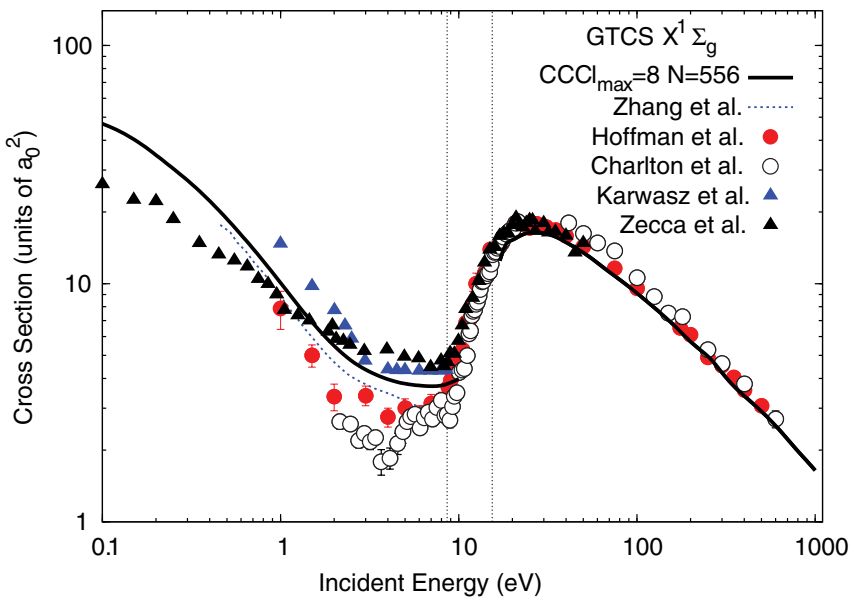

FIG. 2. (Color online) GTCS for positron scattering from the ground state of $\mathrm{H}_{2}$. CCC results are compared with the calculations of Zhang et al. [7] and experiments of Hoffman et al. [23], Charlton et al. [25], Karwasz et al. [28], and Zecca et al. [22].

$l_{\max }=5$, which yield almost identical results to the corresponding CCC calculations (not shown). There is reasonable agreement between the theoretical and experimental results, which show some variation among themselves. At the very lowest energies, we expect that agreement with the experiment of Zecca et al. [22] will improve when the missed scattering to forward angles is accounted for. Above the ionization threshold, there is good agreement between the CCC results and all available experiments [22-27]. Though not shown, there is also good agreement with the first-order calculations of Reid et al. [11].

In Fig. 3, we compare the CCC calculations with the $0.5 \mathrm{eV}$ elastic DCS measured by Sullivan et al. [29]. The experimental setup combines DCS at $\theta$ and $180^{\circ}-\theta$, and we do the same for comparison. The CCC calculations have good convergence by $l_{\max }=8$, and demonstrate the importance of coupling to virtual Ps-formation channels, even for elastic scattering. Comparing with experiment, the CCC results show

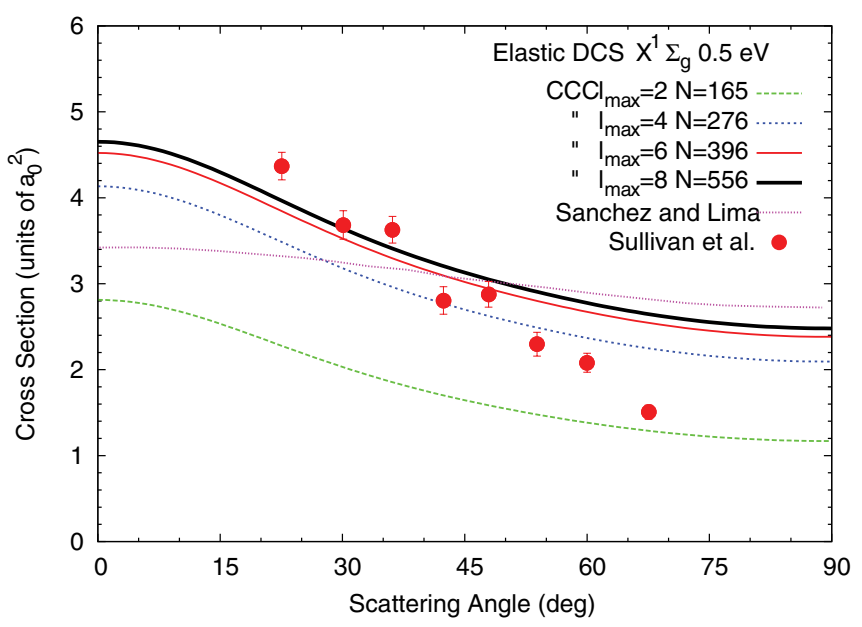

FIG. 3. (Color online) Elastic differential cross sections for positron scattering from the ground state of $\mathrm{H}_{2}$ at an impact energy of $0.5 \mathrm{eV}$. The CCC results (see text) are compared with the calculations of [30] and the measurements of [29].

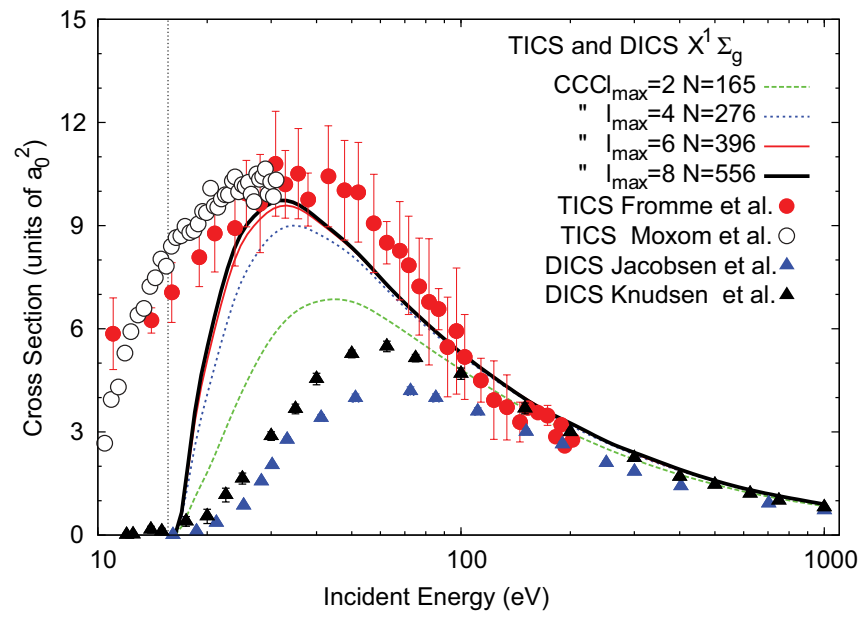

FIG. 4. (Color online) TICS for positron scattering from the ground state of $\mathrm{H}_{2}$. CCC results are compared with the experiments of Fromme et al. [31], Moxom et al. [32], Jacobsen et al. [33], and Knudsen et al. [34].

a substantial improvement on the Schwinger multichannel calculations of Sanchez and Lima [30], particularly at the forward angles. Nevertheless, some discrepancy remains. The rapid rise of the integrated elastic cross section around $0.5 \mathrm{eV}$ (see Fig. 2) is possibly a contributing factor. Otherwise, the lack of account for vibrational and rotational excitations within the present technique may be the reason for the discrepancy.

In the single-center CCC method, the total ionization cross sections (TICS) are calculated as a sum over all positive-energy pseudostate excitation cross sections, and so contain both direct ionization and Ps formation. The present results are compared with the measurements of Fromme et al. [31] and Moxom et al. [32] in Fig. 4. Our TICS results for the largest model $\left(l_{\max }=8\right)$ are in good agreement with experiment at the cross-section maximum $(30 \mathrm{eV})$. At this energy, the Ps-formation cross section is by far the largest contribution compared with the direct ionization cross section (DICS) measured by Jacobsen et al. [33] and Knudsen et al. [34]. The CCC results show a progressively sharper rise just above the ionization threshold as states with larger angular momentum projection are included. As the incident positron energy increases, the Ps-formation cross section diminishes and direct ionization becomes dominant. Similar to electron scattering, the DICS can be accurately described with a CCC model that has smaller values of $l_{\max }$.

In conclusion, we have implemented the $a b$ initio $\mathrm{CCC}$ method for molecular targets, and used positron scattering on molecular hydrogen as an example. Good agreement with experiment from elastic scattering through to total ionization has been demonstrated on a very broad range of energies. The size of the required calculations was found to be particularly large. At low energies, where only elastic scattering is possible, virtual Ps formation needs to be treated to convergence using states with large angular momentum projection. At energies just above the ionization threshold, we need sufficiently many open positive-energy pseudostates to treat the explicit Ps-formation and direct ionization channels. Future work will concentrate on extending the method to positron and electron scattering from more complex molecules. We 
are also planning a formulation that utilizes the spheroidal coordinates, which offers significant advantages for collision processes involving diatomic molecules. Incorporation of an explicit treatment of Ps formation for molecules will also be undertaken.
This work was supported by the Australian Research Council and Curtin University. We are grateful for access to the Australian Partnership for Advanced Computing and its Western Australian node iVEC. Calculations were performed on the EPIC supercomputer funded under the Pawsey Project.
[1] I. Bray, D. V. Fursa, A. S. Kadyrov, A. T. Stelbovics, and A. S. Kheifets, Phys. Rep. 520, 135 (2012).

[2] D. V. Fursa and I. Bray, New J. Phys. 14, 035002 (2012).

[3] I. Bray, D. V. Fursa, A. S. Kheifets, and A. T. Stelbovics, J. Phys. B 35, R117 (2002).

[4] I. Bray and A. T. Stelbovics, Phys. Rev. A 46, 6995 (1992).

[5] I. Bray and D. V. Fursa, Phys. Rev. Lett. 76, 2674 (1996).

[6] I. Bray, Phys. Rev. Lett. 89, 273201 (2002).

[7] R. Zhang, K. L. Baluja, J. Franz, and J. Tennyson, J. Phys. B 44, 035203 (2011)

[8] G. Danby and J. Tennyson, J. Phys. B 23, 1005 (1990).

[9] F. Arretche, R. F. da Costa, S. d. Sanchez, A. N. S. Hisi, E. M. de Oliveira, M. T. d. N. Varella, and M. A. P. Lima, Nucl. Instr. Meth. B 247, 13 (2006).

[10] E. A. G. Armour and J. W. Humberston, Phys. Rep. 204, 165 (1991).

[11] D. D. Reid, W. B. Klann, and J. M. Wadehra, Phys. Rev. A 70, 062714 (2004)

[12] A. S. Kadyrov and I. Bray, Phys. Rev. A 66, 012710 (2002).

[13] R. Utamuratov, A. S. Kadyrov, D. V. Fursa, I. Bray, and A. T. Stelbovics, J. Phys. B 43, 125203 (2010).

[14] A. V. Lugovskoy, A. S. Kadyrov, I. Bray, and A. T. Stelbovics, Phys. Rev. A 82, 062708 (2010).

[15] W. A. Isaacs and M. A. Morrison, Phys. Rev. A 53, 4215 (1996).

[16] T. E. Sharp, At. Data Nucl. Data Tables 2, 119 (1970).

[17] W. Kolos and L. Wolniewicz, J. Chem. Phys. 46, 1426 (1967).

[18] B. H. Bransden, Atomic Collision Theory, Lecture Notes and Supplements in Physics (Benjamin/Cummings, Reading, MA, 1983), 2nd ed.

[19] J.-Y. Zhang, J. Mitroy, and K. Varga, Phys. Rev. Lett. 103, 223202 (2009).
[20] T. Mukherjee and N. K. Sarkar, J. Phys. B 41, 125201 (2008).

[21] W. Tenfen, K. T. Mazon, S. E. Michelin, and F. Arretche, Phys. Rev. A 86, 042706 (2012).

[22] A. Zecca, L. Chiari, A. Sarkar, K. L. Nixon, and M. J. Brunger, Phys. Rev. A 80, 032702 (2009).

[23] K. R. Hoffman, M. S. Dababneh, Y. F. Hsieh, W. E. Kauppila, V. Pol, J. H. Smart, and T. S. Stein, Phys. Rev. A 25, 1393 (1982).

[24] M. Charlton, T. C. Griffith, G. R. Heyland, and G. L. Wright, J. Phys. B 13, L353 (1980).

[25] M. Charlton, T. C. Griffith, G. R. Heyland, and G. L. Wright, J. Phys. B 16, 323 (1983).

[26] A. Deuring, K. Floeder, D. Fromme, W. Raith, A. Schwab, G. Sinapius, P. W. Zitzewitz, and J. Krug, J. Phys. B 16, 1633 (1983).

[27] S. Zhou, H. Li, W. E. Kauppila, C. K. Kwan, and T. S. Stein, Phys. Rev. A 55, 361 (1997).

[28] G. P. Karwasz, D. Pliszka, and R. S. Brusa, Nucl. Instr. Meth. B 247, 68 (2006).

[29] J. P. Sullivan, S. J. Gilbert, J. P. Marler, L. D. Barnes, S. J. Buckman, and C. M. Surko, Nucl. Instr. Meth. B 192, 3 (2002).

[30] S. d. A. Sanchez and M. A. P. Lima, Nucl. Instr. Meth. B 266, 447 (2008).

[31] D. Fromme, G. Kruse, W. Raith, and G. Sinapius, J. Phys. B 21, L261 (1988).

[32] J. Moxom, G. Laricchia, and M. Charlton, J. Phys. B 26, L367 (1993).

[33] F. M. Jacobsen, N. P. Frandsen, H. Knudsen, and U. Mikkelsen, J. Phys. B 28, 4675 (1995).

[34] H. Knudsen, L. Brun-Nielsen, M. Charlton, and M. R. Poulsen, J. Phys. B 23, 3955 (1990). 